\title{
Aspectos da tomografia de coerência óptica na oftalmia simpática: relato de caso
}

\author{
Optical coherence tomography aspects of sympathetic ophthalmia: case report
}

\author{
Maǵno Antônio Ferreira1 \\ Camila Naves Mendonça ${ }^{2}$ \\ Wanderlei Ferreira de Sá Filho ${ }^{3}$ \\ Adriano Silva Neves ${ }^{4}$
}

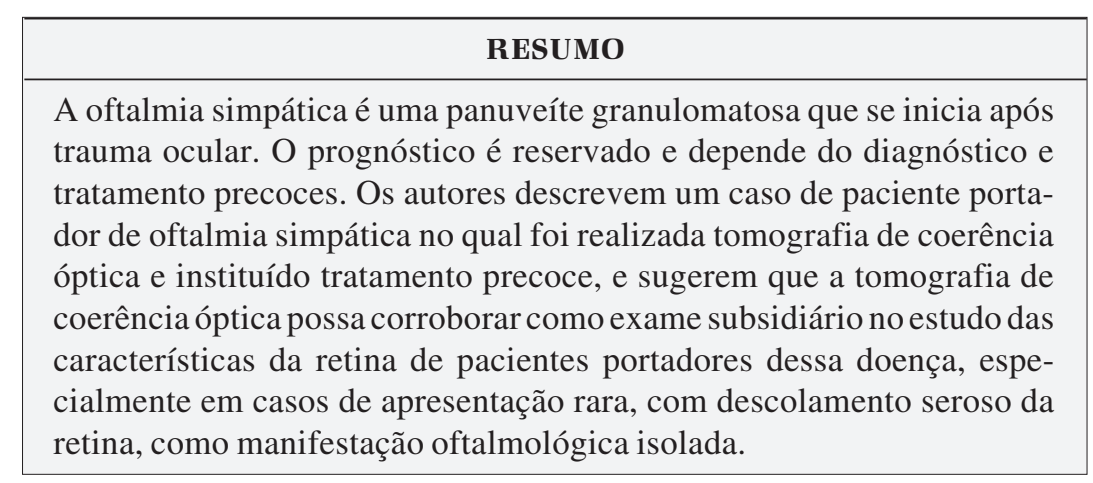

Descritores: Descolamento retiniano; Oftalmia simpática/terapia; Tomografia de coerência óptica; Traumatismos oculares [Tipo de publicação]
Trabalho realizado no Hospital das Clínicas da Faculdade de Medicina da Universidade Federal de Uberlândia - UFU - Uberlândia (MG) - Brasil.

Professor de Oftalmologia da Faculdade de Medicina da Universidade Federal de Uberlândia - UFU - Uberlândia (MG) - Brasil.

${ }^{2}$ Médica do Serviço de Oftalmologia do Hospital das Clínicas da UFU - Uberlândia (MG) - Brasil.

${ }^{3}$ Médico do Serviço de Oftalmologia do Hospital das Clínicas da UFU - Uberlândia (MG) - Brasil.

${ }^{4}$ Acadêmico da Faculdade de Medicina da UFU - Uberlândia (MG) - Brasil.

Endereço para correspondência: Adriano Silva $\mathrm{Ne}-$ ves. Avenida João Pessoa, 794 - Apto. 401 - Uberlândia (MG) CEP 38400-338

E-mail: asneves2003@yahoo.com.br

Recebido para publicação em 25.06.2008 Aprovação em 06.02.2009

\section{INTRODUÇ̃̃OO}

A oftalmia simpática é uma panuveíte granulomatosa bilateral que se inicia após injúria na úvea de um dos olhos ${ }^{(1-2)}$. Na quase totalidade dos casos, o dano uveal é de origem traumática ou cirúrgica. O olho traumatizado é denominado simpatogênico e o olho contralateral de simpatizado. A histopatologia da doença mostra nódulos ou infiltrados difusos de linfócitos e macrófagos no tecido uveal ${ }^{(3)}$. A neovascularização coroidiana, embora raramente associada à oftalmia simpática, pode ocorrer e comprometer o prognóstico visual de um olho já debilitado(4).

O diagnóstico geralmente é realizado por meio da anamnese, com relato de trauma ou cirurgia prévios, pela fundoscopia e com a angiofluoresceinografia, mostrando, nos casos complicados, edema da cabeça do nervo óptico, neovascularização e descolamento de retina ${ }^{(2)}$. As indicações e o momento da enucleação ocular nos casos de oftalmia simpática ainda permanecem controversos ${ }^{(5)}$. Os corticosteróides têm papel fundamental e central no tratamento, com necessidade de altas doses nas primeiras semanas de terapia e monitoramento constante dos efeitos sistêmicos, apresentando reflexos sobre as consequências visuais da oftalmia simpática ${ }^{(2,6)}$.

\section{RELATO DE CASO}

Identificação: GRR, 22 anos, masculino, compareceu ao Serviço de Oftalmologia da Universidade Federal de Uberlândia em março de 2006, com história de trauma facial por agressão física há sete dias.

Ao exame oftalmológico, apresentava acuidade visual de 20/20 (tabela de Snellen) no olho direito e ausência de percepção luminosa com extensa laceração corneoescleral, exposição de tecido uveal e hifema total no olho 
esquerdo. O paciente foi encaminhado ao centro cirúrgico, sendo constatada ruptura traumática grave do globo ocular, e realizada evisceração. No vigésimo primeiro dia de pósoperatório, o paciente retornou queixando-se de embaçamento visual progressivo no olho direito, com acuidade visual de 20/200 e exame de fundo de olho mostrando descolamento seroso de retina em todo o polo posterior (Figura 1A).

A angiofluoresceinografia mostrou múltiplos pontos de vazamentos perimaculares com aspecto característico de "pin point" e a tomografia de coerência óptica (OCT) registrou extensa área de descolamento seroso retiniano (Figura 2A). O paciente recebeu $140 \mathrm{mg} / \mathrm{dia}$ de corticosteróide (Meticorten ${ }^{\circledR}$ ), com redução gradual (10 mg/semana), evoluindo com melhora importante da acuidade visual e do descolamento retiniano já na primeira semana de tratamento. Com 35 dias de corticoterapia, apresentava acuidade visual de 20/25 parcial e resolução completa do descolamento de retina (Figura 2B). A retinografia após 60 dias de tratamento está representada na figura 1 $\mathrm{B}$, evidenciando melhora substancial.

\section{DISCUSSÃO}

A oftalmia simpática é uma panuveíte bilateral, rara, e potencialmente devastadora das funções visuais, tipicamente surgindo após um trauma penetrante não-cirúrgico sobre o globo ocular $^{(7)}$. O uso de corticosteróides sistêmicos revolucionou o prognóstico, e uma boa acuidade visual no olho simpatizado pode ser alcançada ${ }^{(7)}$, particularmente nos casos em que o diagnóstico é feito de forma precoce e a terapia antiinflamatória prontamente instituída com a utilização de doses elevadas do medicamento ${ }^{(6)}$.

Estudos têm demonstrado que o uso de drogas imunossupressoras, como ciclosporina e azatioprina, em combinação com esteróides sistêmicos, permitem o controle da inflamação intraocular com uma dose menor de esteróide, com consequente redução dos efeitos gerais que acompanham os esteróides de uso sistêmico ${ }^{(7)}$.

A tomografia de coerência óptica (OCT), apesar de descrita há mais de dez anos, tem emergido como uma nova técnica que, através de imagens de alta resolução de cortes seccionais da retina, pode auxiliar no diagnóstico, na monitorização e na análise quantitativa de doenças que acometem a mácula e a cabeça do nervo óptico ${ }^{(8-10)}$. No caso descrito anteriormente, realizamos a OCT precocemente, evidenciando edema e descolamento seroso da retina. Após 35 dias de tratamento, pudemos acompanhar a resolução do quadro através de nova OCT, corroborando dados sugestivos de prognóstico favorável dos casos de oftalmia simpática submetidos a tratamento médico precoce ${ }^{(10)}$.

Dessa forma, oftalmologistas devem estar cientes da variedade de características morfológicas das diferentes formas de uveítes que podem ser observadas com o uso da OCT. Esse reconhecimento pode auxiliar no diagnóstico precoce, que deve ser sempre precedido de uma boa anamnese, exame de fundo de olho com o oftalmoscópio binocular indireto e a angiofluoresceinografia ${ }^{(9)}$. Além disso, a OCT sugere ser útil no acompanhamento dos pacientes em tratamento do edema macular ocasionado por uveítes ${ }^{(8)}$.

\section{CONCLUSÃO}

Demonstramos um caso de oftalmia simpática, no qual a OCT, atuando como método coadjuvante, auxiliou no diagnóstico precoce da doença, permitindo analisar com mais detalhes as alterações retinianas, sobretudo na região macular. $\mathrm{O}$ tratamento com corticosteróide foi instituído precocemente, obtendo-se excelente resultado anatômico e funcional.
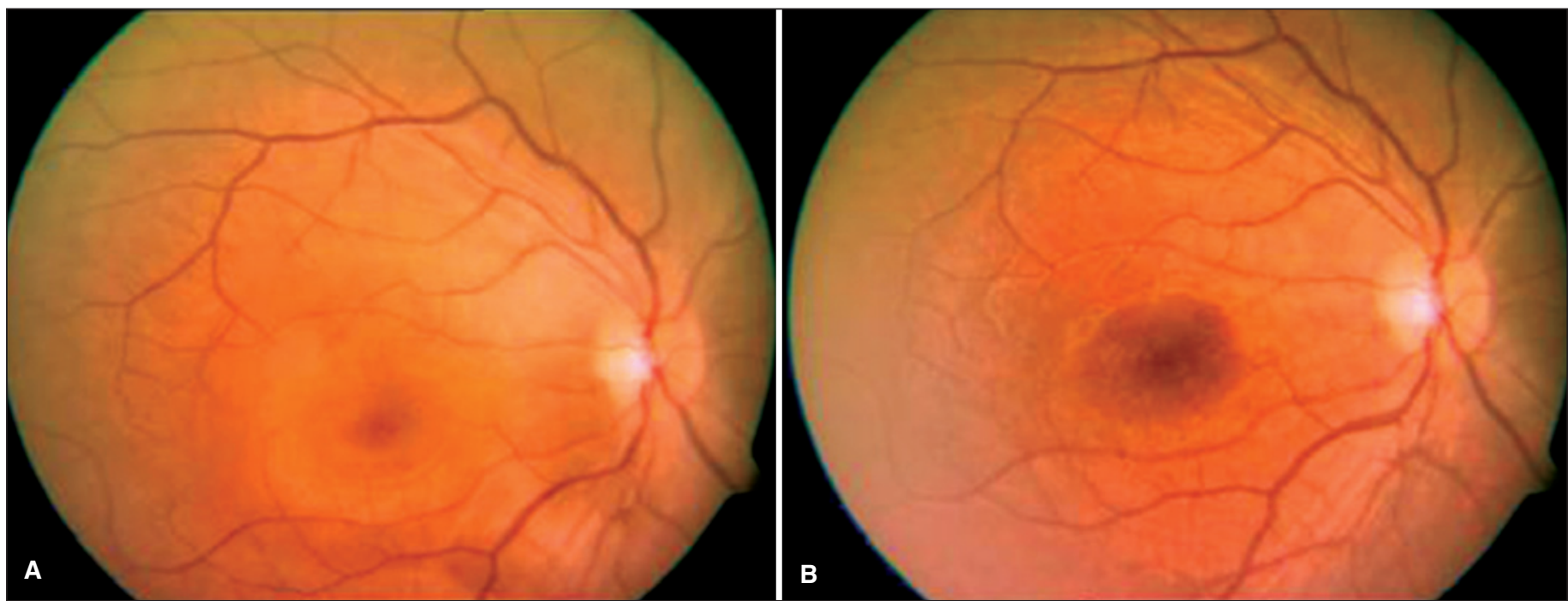

Figura 1 - Retinografia do olho direito. A) Pré-tratamento (nota-se descolamento seroso da retina no polo posterior, comprometendo toda região macular); B) Após 60 dias de tratamento (resolução completa do descolamento). 

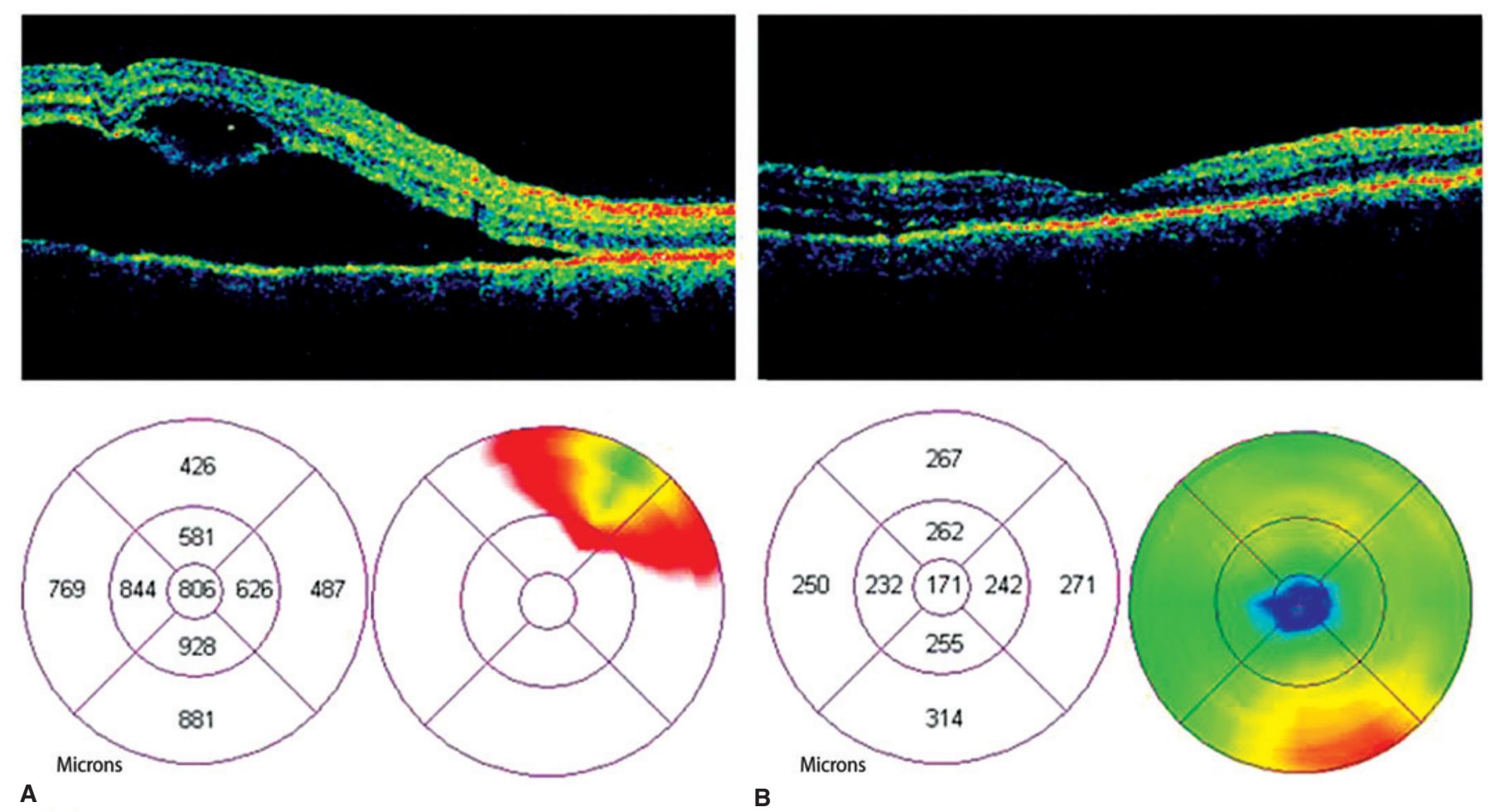

Figura 2 - Tomografia de coerência óptica (OCT) do olho direito. A) Pré-tratamento (verifica-se descolamento seroso da retina no polo posterior comprometendo a fóvea, com pequena área cística subfoveal e aumento da espessura retiniana em todos os quadrantes, sendo de 806 micra na regiẫo central); B) Após 35 dias de tratamento (notamos desaparecimento do descolamento seroso da retina, com espessura da área central de 171 micra).

\section{ABSTRACT}

The sympathetic ophthalmia is a granulomatous panuveitis that starts after ocular trauma. The prognosis is poor and depends of both early diagnosis and treatment. The authors describe one case of a patient with sympathetic ophthalmia using optical coherence tomography who was submitted to early treatment, and it suggests that the optical coherence tomography can corroborate as a ancillary method in the study of retina characteristics in sympathetic ophthalmia disease specially in cases of rare presentation, with serous retinal detachment as an isolated ophthalmological manifestation.

Keywords: Retinal detachment; Ophthalmia, sympathetic/therapy; Tomography, optical coherence; Eye injuries [Publication type]

\section{REFERÊNCIAS}

1. Chan CC, Roberge FG. Sympathetic ophthalmia. In: Pepose JS, Holland GN, Wilhelmus KR, editors. Ocular infection and immunity. St. Louis: Mosby; 1996. p.723-33.
2. Chan CC, Mochizuki M. Sympathetic ophthalmia: an autoimmune ocular inflammatory disease. Springer Semin lmmunopathol. 1999;21(2):125-34.

3. Fuchs E. Über sympathisierende Entzundung zuerst Bemerkungen über seröse traumatische Iritis. Albrecht Von Graefes Arch Ophthalmol. 1905;61: 365-456.

4. Vianna RN, Özdal P, Souza Filho JP, Deschênes J. [Choroidal neovascularization associated with sympathetic ophthalmia: case report]. Arq Bras Oftalm. 2005;68(3):397-400. Portuguese.

5. Kilmartin DJ, Dick AD, Forrester JV. Prospective surveillance of sympathetic ophthalmia in the UK and Republic of Ireland. Br J Ophthalmol. 2000;84(3): 259-63. Comment in: Br J Ophthalmol. 2004;88(3):438.

6. Chan CC, Roberge RG, Whitcup SM, Nussenblatt RB. Thirty-two cases of sympathetic ophthalmia. A retrospective study at the National Eye Institute, Bethesda, Md., from 1982 to 1992. Arch Ophthalmol. 1995;113(5):597-600. Erratum in: Arch Ophthalmol. 1995;113(12):1507.

7. Vote BJ, Hall A, Cairns J, Buttery R. Changing trends in sympathetic ophthalmia. Clin Experiment Ophthalmol. 2004;32(5):542-5.

8. Reinthal EK, Völker M, Freudenthaler N, Grub M, Zierhut M, Schlote T. [Optical coherence tomography in the diagnosis and follow-up of patients with uveitic macular edema]. Ophthalmologe. 2004;101(12):1181-8.

9. Gallagher MJ, Yilmaz T, Cervantes-Castañeda RA, Foster CS. The characteristic features of optical coherence tomography in posterior uveitis. Br J Ophthalmol. 2007;91(12):1680-5.

10. Markomichelakis NN, Halkiadakis I, Pantelia E, Georgalas E, Chrysanthi K, Theodossiadis $\mathrm{P}$, et al. Course of macular edema in uveitis under medical treatment. Ocul Immunol Inflamm. 2007;15(2):71-9. Erratum in: Ocul Immunol Inflamm. 2007;15(4):369. Anthi, Koutsandrea [corrected to Chrysanthi, Koutsandrea]. Ocul Immunol Inflamm. 2008;16(5):251. Georgalas, Elias [corrected to Georgalas, Ilias]. 